\title{
Methyltrioxorhenium catalysed synthesis of highly oxidised aryltetralin lignans with anti-topoisomerase II and apoptogenic activities
}

\author{
Raffaele Saladino, ${ }^{\mathrm{a}, \mathrm{b}, *}$ Cinzia Fiani, ${ }^{\mathrm{b}}$ Maria Cristina Belfiore, ${ }^{\mathrm{b}}$ Giampiero Gualandi, \\ Sabrina Penna ${ }^{\mathrm{b}}$ and Pasquale Mosesso ${ }^{\mathrm{b}}$ \\ ${ }^{a}$ INFM, della Tuscia, via S.Camillo De Lellis, I-01100 Viterbo, Italy \\ ${ }^{\mathrm{b}}$ Dipartimento A.B.A.C., Università della Tuscia, via S.Camillo De Lellis, I-01100 Viterbo, Italy
}

Received 10 March 2005; revised 1 July 2005; accepted 8 July 2005

Available online 19 August 2005

\begin{abstract}
A novel and efficient procedure to prepare highly oxidised aryltetralin lignans, such as isopodophyllotoxone and (-)-aristologone derivatives, by oxidation of podophyllotoxin and galbulin with methylrhenium trioxide (MTO) and novel MTO heterogeneous catalysts is reported. It is noteworthy that in the case of isopodophyllotoxone derivatives the functionalisation of the C-4 position of the C-ring and the ring-opening of the D-lactone moiety increased the activity against topoisomerase II while causing the undesired inhibition of tubulin polymerisation to disappear. The novel (-)-aristologone derivatives showed apoptogenic activity against resistant human lymphoma cell lines.

(c) 2005 Elsevier Ltd. All rights reserved.
\end{abstract}

\section{Introduction}

Lignans are a family of natural products with a broad variety of biological and pharmacological activities. ${ }^{1}$ Among them, aryltetralin derivatives are of special interest owing to their powerful antitumoral, antimitotic, antiviral, cardiovascular and immunosuppressive activity. ${ }^{2}$ Aryltetralin derivatives show also a selective nonredox inhibition of 5-lipoxygenase by interaction with the arachidonic acid binding site and could form a new class of therapeutic agents for the treatment of asthma and rheumatoid arthritis. ${ }^{3}$ Podophyllotoxin $\mathbf{1}$, isolated from different plants of the genus Podophyllum, is the most investigated aryltetralin derivative. It is a well-established inhibitor of cell division at the level of the microtubule assembly by freezing polymerisation of tubulin at the colchicine site. ${ }^{4}$ This activity has led to the design of semi-synthetic derivatives, such as etoposide (4'-demethyl-7-[4,6- $O$-ethylidene- $\beta$-Dgluco-pyranosyl epipodophyllotoxin) and teniposide (4'-demethyl-7-[4,6- $O$-thenilidene- $\beta$-D-glucopyranosyl epip-

\footnotetext{
Keywords: Lignans; Oxidative functionalisation; Methyltrioxorhenium; Topoisomerase II; Apoptogenic activity.

* Corresponding author. Tel.: + 3907613572 84; fax: + 390761357 242; e-mail: saladino@unitus.it
}

odophyllotoxin), which have been widely used for the treatment of small-cell lung cancer, testicular cancer, lymphoma and acute lymphocytic leukaemia. ${ }^{5}$ Etoposide and teniposide show different side effect profiles compared to podophillotoxin due to their action as selective inhibitors of DNA topoisomerase II, a key enzyme involved in DNA transcription, replication, recombination and possibly DNA repair. ${ }^{6}$ In recent years, several syntheses of podophyllotoxin and aryltetralin lignan derivatives have been reported in the literature which mainly focused on structural modifications of leader molecules to obtain less toxic analogues with high biological activities. On the other hand, a few data are available on oxidative functionalisation, ${ }^{7}$ a process that plays a relevant role in their biological mechanism of action. For example, it is known that etoposide undergoes oxidative orthodemethylation by a cytochrome P450-dependent metabolic process to a $3^{\prime}, 4^{\prime}$-dihydroxy derivative. ${ }^{8}$ This derivative is further oxidised to the corresponding ortho-benzoquinone, a highly reactive intermediate responsible for the irreversible binding to proteins and DNA. ${ }^{9}$ In a similar way, a semibenzoquinone free radical intermediate of etoposide is responsible for DNA strand breakage. ${ }^{10}$ Since general and selective methods for the oxidation of aryltetralin lignans are still lacking, ${ }^{11}$ novel synthetic strategies are needed to 